\author{
Military Technical College \\ Kobry El-Kobbah, \\ Cairo, Egypt
}

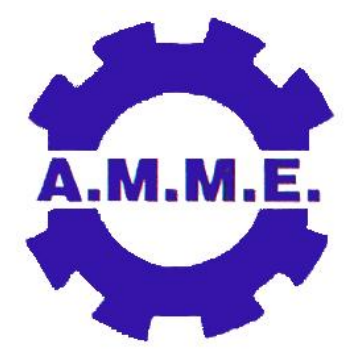

14th International Conference on Applied Mechanics and Mechanical Engineering.

\title{
Effect of elastic modulus variation during plastic deformation on simulations of uniaxial ratchetting
}

By

\author{
M. Abdel-Karim*
}

\begin{abstract}
:
The objective of the present work is to highlight the effect of variation of the elastic modulus during plastic deformation on uniaxial ratchetting simulations. In order to achieve this target, the variation of the elastic modulus during loading and un-loading is considered and discussed based on some experimental observations which pointed out recently. Then the effect of such variation on simulations is scrutinized from the theoretical point of view by considering simulations of ratchetting experiments conducted on stainless steel 304L using the well-known Armstrong-Frederick model. It is shown that, using two different values for the elastic modulus during loading and un-loading could have a significant effect on simulations of uniaxial ratchetting. The importance of such findings is that the excessive ratchetting over-prediction resulting from any specific kinematic hardening rule is expected to decrease significantly by taking into consideration this effect. In this case, it is necessary to include variation of elastic modulus within the scheme of constitutive modeling of cyclic plasticity to simulate actual material responses especially ratchetting.
\end{abstract}

\section{Keywords:}

Constitutive modeling, cyclic plasticity, ratchetting, kinematic hardening, 304 stainless steel and elastic modulus 
Proceeding of the $14^{\text {th }}$ AMME Conference, $25-27$ May 2010

* College of Engineering, Fayoum University, Fayoum, Egypt 


\section{Introduction:}

Throughout the last three decades, numerous studies have attempted to determine the controlling and fundamental factors that affecting ratchetting and to find appropriate ways to replicate these factors into the scheme of constitutive modeling to be able to simulate experimental responses as accurate as possible. To obtain a better appreciation for this phenomenon, i.e. ratchetting, it is worth to note that ratchetting is concerned with secondary deformation accumulating cycle by cycle in the direction of mean stress, therefore, it is not easy to describe it quantitatively.

During simulations of ratchetting, similar to simulations of other material responses, elastic behaviour is usually assumed to be constant. It is worth to remember that elastic behaviour is the result of atomic bond stretching and it is assumed to be linear because this is a good first order approximation. However, exact application of elastic theory includes higher order terms that lead to a small amount of nonlinear recovery [1]. In addition, different experiments have indicated that during plastic deformation, the elastic modulus $(E)$ might change significantly [2-4]. In this case, it is expected that such variation could have a significant influence on small-scale plasticity as in ratchetting.

Throughout the context of studying different variables that might affect ratchetting, influence of variation of elastic modulus during loading, un-loading and re-loading has not been considered yet, neither experimentally or theoretically. In simulations and within the scheme of constitutive models that replicate ratchetting responses, it is common during plastic deformation assuming constant and identical values for the elastic modulus throughout loading, un-loading and re-loading. However, as aforementioned, different experiments indicated that during plastic deformation, the value of the elastic modulus might change significantly. In this case, such change could have a considerable effect on simulation of ratchetting especially uniaxial ratchetting, since it deals with small accumulation of plastic strain cycle by cycle. This point has not been studied yet. Therefore, it is highly recommended to examine and scrutinize the influence of variation of the elastic modulus during plastic deformation on overall ratchetting simulations, which is the aim of this work.

\section{Variation of elastic modulus during plastic deformation:}

In fact, only some experiments have been conducted to study the change of elastic modulus during loading and unloading. Initially, Taylor and Quinney [5] pointed out with no extensive details the change of Young's Modulus during loading un-loading curves based on experiments on annealed tubes of aluminum. Then during the last two decades a considerable effort has been focused to explore this point in details.

First, in 1996 Morestin and Boivin [6] focused experimentally on evolution of Young's modulus on the basis of plastic strains between $0 \%$ and $15 \%$ for steel. They considered plain steel and alloys A33, XC38 and AU4G with a large cross-section and sheet metal type XE280D with a small cross-section. Accordingly, Morestin and Boivin pointed out a significant decrease in Young's modulus (about 10\% of the initial value after only 5\%) for these materials. Next, in 1997, Ishikawa [7] investigated the subsequent yield surfaces after tension and/or torsion preloading for an initially isotropic material of SUS304. Ishikawa observed a significant change in Young's modulus between initial loading and unloading curve even for relatively small prestrain value. However, a small change is observed between initial loading and re-loading curves. Then, Ghosh and his co-workers [2-3] scrutinized spring back strain or 
compliance after uniaxial stretching of the sheet metal (a BCC high strength steel alloy (GP50XK60 and DQSK steel) and an FCC automotive aluminum alloy (6022-T4 Al )) to different levels of prestrain. In this case also, Ghosh and his co-workers indicated the variation of the average moduli calculated at different levels of prestrain for high strength steel and for the aluminum alloy, where increasing levels of plastic strain result in a lower effective modulus. After that, Zhou et al. [8] characterized the cyclic plasticity behaviour of nickel single crystals oriented for single slip by uniaxial, symmetric, tension-compression, strain controlled tests carried out at constant plastic strain amplitudes ranging from $5\left(10^{-5}\right)$ to $1\left(10^{-3}\right)$. Based on these experiments, Zhou and his-coworkers pointed out a significant variation for Young's modulus with accumulated plastic strain.

Finally, Khan and his co-workers [4] evaluated experimentally the initial and subsequent yield surfaces in Al 6061-T6511. In these experiments, they showed that, for Al 6061-T6511, there was about $5 \%$ decrease in the value of Young's modulus with plastic deformation in the case of tensile loading. Consequently, and based on the aforementioned different experimental facts and observations, it can be deduced that, for some materials Young's modulus is not constant during plastic deformation throughout loading, unloading and reloading. Therefore, this experimental fact should be considered during simulations because it might have a significant effect on numerical results. In addition, it could totally change the outcome from the models.

\section{Kinematic hardening rule:}

In this work, the objective is not to simulate ratchetting, but it is merely to show the influence of variation of Young's modulus on overall simulations of ratchetting. Therefore, the wellknown hardening rule of Armstrong and Frederick is considered. This specific rule is chosen because it conservatively over predicts uniaxial ratchetting, but able to replicate different material behaviours [9]. Armstrong and Frederick [10] proposed the simplest nonlinear kinematic hardening rule by introducing the concepts of strain hardening and dynamic recovery that construct the kinematic hardening rule nonlinear in nature. The initial Armstrong-Frederick rule was given as:

$$
\mathrm{d} \underset{\sim}{\alpha}=\zeta\left(\frac{2}{3} r \mathrm{~d} \boldsymbol{\varepsilon}^{p}-\underset{\sim}{\alpha} \mathrm{d} p\right)
$$

where $\zeta$ and $r$ are material hardening parameters, $\mathrm{d} p$ refers to accumulated plastic strain increment:

$$
\mathrm{d} p=\sqrt{(2 / 3) \mathrm{d} \boldsymbol{\varepsilon}^{p} \bullet \mathrm{d} \varepsilon_{\sim}^{p}}
$$

Chaboche et al. [11] introduced the concept of decomposition of hardening variable into several components and proposed a multiple nonlinear kinematic hardening rule based on the initial Armstrong-Frederick rule where:

$$
\mathrm{d} \underset{\sim}{\alpha}=\sum_{i=1}^{M} \mathrm{~d}{\underset{\alpha}{i}}_{i}
$$




$$
\mathrm{d} \alpha_{i}=\zeta_{i}\left(\frac{2}{3} r_{i} \mathrm{~d} \varepsilon^{p}-\alpha_{i} \mathrm{~d} p\right)
$$

This non-linear kinematic hardening rule simulates well various uniaxial material responses but it still over-predicts uniaxial as well as multiaxial ratchetting responses [9, 12-13]. As a basic modification to the Armstrong-Frederick non-linear kinematic hardening rule, Ohno and Wang [14], introduced a power function in each decomposed hardening rule as:

$$
\mathrm{d} \alpha_{i}=\zeta_{i}\left((2 / 3) r_{i} \mathrm{~d} \varepsilon_{\sim}^{p}-\left(\bar{\alpha}_{i} / r_{i}\right)^{m_{i}} \mathrm{~d} p \underset{\sim}{\alpha_{i}}\right)
$$

This equation has been also examined in simulating ratchetting and it has been established that, the outcome depends mainly on the value of the material parameter $m_{i}$, where the higher the value $m_{i}$, the lower the ratchetting strain. Equation (5) will be called as the Armstrong-Frederick model from now on through out this work.

Determinations of the different material parameters defined in Eq. (5) have a systematic sequence. The main parameters $\left(\zeta_{i}, r_{i}\right)$ can be determined by using a very large value for $m_{i}\left(m_{i} \rightarrow \infty\right)$. In this case, under uniaxial tensile, Eq (5) reduces to the common OhnoWang rule [15] and only monotonic tensile curve is essential to determine these parameters. Further details regarding determination for these parameters $\left(\zeta_{i}, r_{i}\right)$ can be found elsewhere [13]. On the other hand, value of $m_{i}$ is decided to simulate uniaxial ratchetting experiments as accurate as possible.

\section{Constitutive Modeling:}

In this work, we consider only small strain range. Thus, the total strain $\underset{\sim}{\varepsilon}$ is the sum of the elastic part, $\mathcal{\varepsilon}^{e}$, and the plastic part, $\mathcal{\varepsilon}^{p}$. With elastic constants $E$ and $v$, we have:

$$
\begin{aligned}
& \underset{\sim}{\boldsymbol{\varepsilon}}={\underset{\sim}{\boldsymbol{\varepsilon}}}^{e}+{\underset{\sim}{\boldsymbol{\varepsilon}}}^{p} \\
& \boldsymbol{\varepsilon}^{e}=\frac{1+v}{E} \underset{\sim}{\sigma}-\frac{v}{E} \operatorname{tr}(\underset{\sim}{\sigma}) \underset{\sim}{\mathbf{I}}
\end{aligned}
$$

where $\underset{\sim}{\sigma}$ indicates stress tensor, tr the trace, $\underset{\sim}{\mathbf{I}}$ signifies the unit tensor of rank two.

In this work, no cyclic hardening is considered though austenitic stainless steels exhibit significant cyclic hardening. However, such assumption could be acceptable under uniaxial ratchetting especially if the strain range is small [16]. In addition, through out the simulations isotropic hardening and the rate effect are neglected so as to discuss explicitly the effect of variation of the elastic modulus. Especially, cyclic hardening for AISI 304 stainless can be attributed to kinematic hardening not isotropic hardening [17]. Thus, the yield function can be expressed according to von Miss as:

$$
\boldsymbol{F}=\frac{3}{2}(\underset{\sim}{\boldsymbol{s}}-\underset{\sim}{\boldsymbol{\alpha}}):(\underset{\sim}{\boldsymbol{s}}-\underset{\sim}{\boldsymbol{\alpha}})-\sigma_{o}^{2}
$$


Where $\underset{\sim}{\boldsymbol{s}}=\underset{\sim}{\sigma}-(1 / 3) \underset{\sim}{\boldsymbol{\delta}}$ is the component of the deviatoric stress tensor, $\underset{\sim}{\boldsymbol{\alpha}}$ represents the component of the deviatoric back stress, and $\sigma_{o}$ is the tensile yield stress. Furthermore, the plastic strain rate, $\dot{\sim}^{p}$, assumed as:

$\dot{\boldsymbol{\varepsilon}}^{p}=\frac{1}{h}\langle\underset{\sim}{\dot{s}}: \underset{\sim}{\boldsymbol{n}}\rangle \boldsymbol{\sim}$

Where $\langle x\rangle=0.5(x+|x|), \underset{\sim}{\boldsymbol{n}}$ is the components of the unit exterior normal on the yield surface at the loading point, and $h$ is the plastic modulus function.

\section{Simulations of Experiments and Discussion:}

Uniaxial ratchetting experiments conducted on stainless steel SS304L by Hassan et al. [18] are considered as a benchmark test in this work. Simulations using the Armstrong-Frederick model with constant value for the elastic modulus, $E$, during loading and different values during unloading are compared to these experimental responses. In this experiment, the mean or steady axial stress $\left(\sigma_{m}\right)$ prescribed was $50 \mathrm{MPa}$, whereas the equivalent amplitude stress, $\sigma_{e q}=\sqrt{\left(\sigma-\sigma_{m}\right)^{2}+3 \tau^{2}}$, presented by the radius of the dotted circle in Fig. 1, was kept $200 \mathrm{MPa}$.

Now let us discuss the influence of elastic modulus on simulations of the aforementioned ratchetting experiment using the Armstrong-Frederick model. Again, it is worth to remember that the aim is not to fit experiment, but it is mainly to discuss the effect of variation of elastic modulus on ratchetting simulations. To achieve this target, classical results, in which only one and identical value for the elastic modulus $(E)$ is implemented in calculations during loading and unloading, will be compared with the results in which two different values for the elastic modules are considered. These two different values are taken based on the experimental results of Ishikawa [7] and listed with the necessary material parameters for the constitutive model in Table 1. One might here argue that these two values for the elastic modulus are not same as those values for the material used in the considered experiments of Hassan et al. [18]. In order to verify this argument, it should be noted that, Ishikawa conducted his experiments on 304 stainless steel as well. Moreover, in this work merely the overall tendency is required to explore the effect of variation, then exact values that necessitate more additional experiments can be recommended to be calculated and utilized. 



loading: $0-1-2$; un-loading: 2-1-3; re-loading: $3-1-2$

Figure (1): loading path and history in uniaxial ratchetting experiment

The simulations by the Armstrong-Frederick model for the uniaxial ratchetting are shown in Fig. 2. It is worth to note in this figure the amount by which ratchetting is over predicted by the Armstrong-Frederick model as long as constant and similar value for the elastic modulus $(E)$ is considered. On the other hand, this over-predicted ratchetting is exclusively reduced when different values for the elastic modulus during loading and unloading are implemented in calculations. To clarify this statement, let us consider Fig. 3 in which variation of stress versus strain hysteresis loop during the first cycle is illustrated in the two cases; i.e. identical and different values for the elastic modulus. It is clear that, when the value of the elastic modulus $(E)$ is assumed to be constant and identical during loading and unloading, the strain point after re-loading and before unloading for the second cycle is larger than that value in the other case in which the value of the elastic modulus is dissimilar. This effect provides thinner stress strain hysteresis loops with increasing loading cycles and as a result total predicted ratchetting strain will decrease, as shown in Fig. 4.

Table (1): Material parameters for SS $304 \mathrm{~L}$ at room temperature

\begin{tabular}{|l|l|l|}
\hline Elastic constants & $\sigma_{o}=153.2, v=0.298$, \\
\hline Basic kinematic & $r_{o 1}=18, \quad \zeta_{1}=1 \times 10^{4}$ & $r_{o 5}=5.5, \quad \zeta_{5}=8.3 \times 10^{2}$ \\
hardening parameters & $r_{o 2}=12, \quad \zeta_{2}=5 \times 10^{3}$ & $r_{o 6}=6.6, \quad \zeta_{6}=3.4 \times 10^{2}$ \\
$(M=8)$ & $r_{o 3}=8.5, \quad \zeta_{3}=2 \times 10^{3}$ & $r_{o 7}=11, \quad \zeta_{7}=2.5 \times 10^{2}$ \\
& $r_{o 4}=7.2, \quad \zeta_{4}=1.4 \times 10^{3}$ & $r_{o 8}=69.5, \zeta_{8}=5.5 \times 10^{1}$ \\
\hline & During loading and reloading $E=205 \times 10^{9}$ \\
& Elastic modulus & $E=190 \times 10^{9}$ \\
\hline
\end{tabular}






Figure (2): Effect of Elastic modulus on uniaxial ratchetting strain

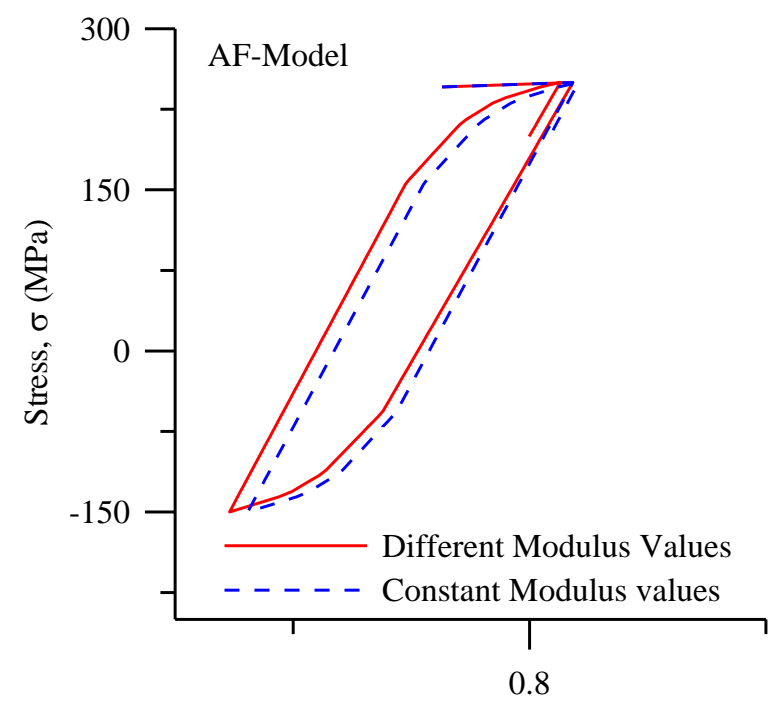

Figure (3): Effect of Elastic modulus on the shape of stress strain hysteresis loop 

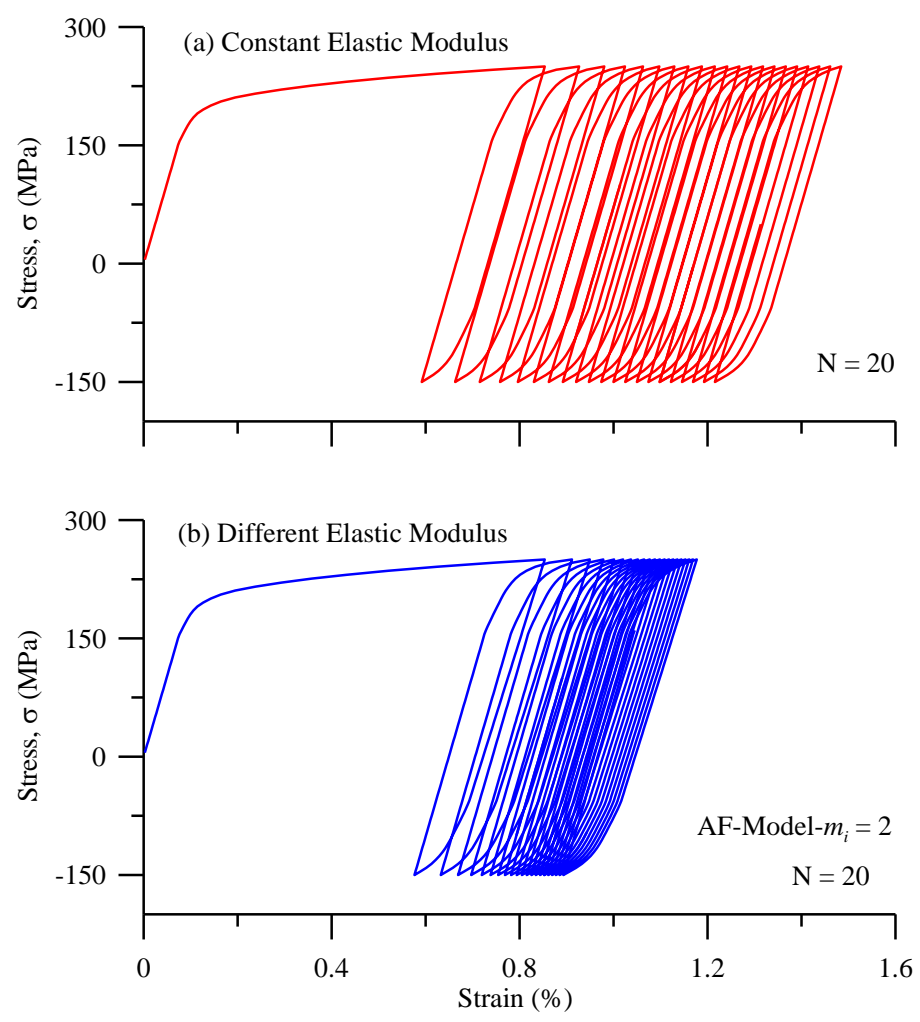

Figure (4): Effect of Elastic modulus on stress-strain hysteresis loops

It is well-known that austenitic stainless steels exhibit significant viscoplasticity at room temperature, as was observed experimentally [12, 16, 19]. In addition, the viscoplasticity influences ratchetting and induces uniaxial ratchetting even under cyclic zero-to-tension loading as explored by Mizuno et al. [16]. Therefore, it should be mentioned that, viscoplasticity needs to be considered for "quantitative" simulations of uniaxial ratchetting of austenitic stainless steel at room temperature $[12,20]$. Therefore, simulations of uniaxial ratchetting shown in Fig. 2 could be enhanced if viscoplasticity effect is taken into consideration.

\section{Conclusions:}

In this work, the effect of elastic modulus variation on simulating uniaxial ratchetting is scrutinized. The findings in this work are concluded mainly based on simulation of uniaxial ratchetting experiments conducted on stainless steel $304 \mathrm{~L}$ within small strain range. It is shown that, in simulating uniaxial ratchetting, it is necessary to consider the variation of elastic modulus during plastic deformation. Using two different but constant values for the elastic modulus $(E)$ during loading and unloading extensively reduces the simulated uniaxial ratchetting strain. Although, the difference of elastic modulus between unloading and reloading can affect the simulated values of uniaxial ratchetting strain by the constitutive model, while such effect is not the main factor we should consider in the construction of constitutive model for cyclic plasticity. In addition, during loading and unloading, two different but constant values are used. On the other hand, some experiments indicated that the value of the elastic modulus could change according to dissimilar governing relations during loading and/or unloading. In this case, it is expected that, simulations of ratchetting will be significantly affected. 


\section{References:}

[1] T. E. Wong and G. C. Johnson., G.C., 1988, On the effects of elastic nonlinearity in metals, Transactions of the ASME, Vol. 110, P. 332-337, 1988.

[2] R. M. Cleveland and A. K. Ghosh, Inelastic effects on springback in metals, International Journal of Plasticity, Vol. 18, P. 769-785, 2002.

[3] L. Luo and A. K. Ghosh, Elastic and inelastic recovery after plastic deformation of DQSK steel sheet, ASME, Journal of Engineering Materials and Technology, Vol. 125, No. 3, P. 237-246, 2003.

[4] A. S. Khan, R. Kazmi, A. Pandey, and T. Stoughton, Evolution of subsequent yield surfaces and elastic constants with finite plastic deformation. Part-I: A very low work hardening aluminum alloy (Al6061-T6511), International Journal of Plasticity Vol. 25, P. 1611-1625, 2009.

[5] G. I. Taylor and H. Quinney, The plastic distortion of metals. Phil. Trans. R. Soc., 230A, P. 323, 1931.

[6] F. Morestin and M. Boivin, On the necessity of taking into account the variation in the Young modulus with the plastic strain in elastic-plastic software, Nuclear Engineering and Design, Vol. 162, No. 1, P. 107-116, 1996.

[7] H. Ishikawa, Subsequent yield surface probed from its current center, International Journal of Plasticity, Vol. 13, No. 6-7, P. 533-549, 1997.

[8] D. Zhou, J. C. Moosbrugger, Y. Jia and D. J. Morrison, A substructure mixtures model for the cyclic plasticity of single slip oriented nickel single crystal at low plastic strain amplitudes, International Journal of Plasticity, Vol. 21, No. 12, P. 2344-2368, 2005.

[9] J. L. Chaboche, A review of some plasticity and viscoplasticity constitutive theories, International Journal of Plasticity, Vol. 24, P. 1642-1693, 2008.

[10] P. J. Armstrong and C. O. Frederick, A mathematical representation of the multiaxial bauschinger effect, CEGB Report RD/B/N731, Berkeley Nuclear Laboratories, Berkeley, UK, 1966.

[11] J. L. Chaboche, K. Dang-Van and G. Cordier, Modelization of the strain memory effect on the cyclic hardening of 316 stainless steel, $5^{\text {th }}$ Int. Conf. on SMiRT, L, Berline, Germany, 1979

[12] G. Z. Kang, Ratchetting: Recent progresses in phenomenon observation, constitutive modeling and application. International Journal of Fatigue, Vol. 30, No. 8, P. 14481472, 2008.

[13] M. Abdel-Karim, Modified kinematic hardening rules for simulations of ratchetting, International Journal of Plasticity, Vol. 25, P. 1560-1587, 2009.

[14] N. Ohno and J-D. Wang, Kinematic hardening rules for simulation of ratchetting behavior, European Journal of Mechanics A/Solids, Vol. 13, No. 4, P. 519-531, 1994.

[15] N. Ohno and J-D Wang, Kinematic hardening rules with critical state of dynamic recovery, International Journal of Plasticity, Vol. 9, No. 3, P. 375-390, 1993.

[16] M. Mizuno, Y. Mima, M. Abdel-Karim and N. Ohno, Uniaxial ratchetting of 316FR steel at room temperature, Part I - Experiments, ASME Journal of Engineering Materials and Technology, Vol. 22, No. 1, P. 29-34, 2000.

[17] H. C. Wu and W. C. Yeh, On the experimental determination of yield surfaces and some results of annealed 304 stainless steel, International Journal of Plasticity, Vol. 7, No. 8, P. 803-826, 1991. 
[18] T. Hassan, L. Taleb and S. Krishna, Influences of non-proportional loading paths on ratcheting responses and simulations by two recent cyclic plasticity models, International Journal of Plasticity, Vol. 24, P. 1863-1889, 2008.

[19] E. Krempl, An experimental study of room-temperature rate-sensitivity, creep and relaxation of AISI type 304 stainless steel, Journal of the Mechanics and Physics of solids, Vol. 27, No. 5/6, P. 363-375, 1979,

[20] N. Ohno and M. Abdel-Karim, Uniaxial ratchetting of 316FR steel at room temperature, Part II - Constitutive modeling and simulation, ASME Journal of Engineering Materials and Technology, Vol. 22, No. 1, P. 35-41, 2000. 